quarters of the year there was no change in the frequency (\%) of oxygenation assessment $(96.0,96.1,97.6,98.8)$, initial antibiotic selection $(81.9,77.9,80.9,83.7)$, or initial antibiotic within $6 \mathrm{~h}(67.1$, $64.0,63.6,63.5)$ or blood cultures performed before antibiotics (58.8, $56.3,57.9,62.4)$ but a small rise in smoking cessation advice (36.4, $32.6,40.3,45.5)$. Overall score, whether composite or appropriate did not change.

Individual trusts Of the 22 Trusts with complete results, overall score over the year was $<80 \%$ in one, $80-85 \%$ in two, $85-90 \%$ in three, $90-95 \%$ in 13 and $>95 \%$ in three. Comparing last with first quarter scores only five individual Trusts showed a rise in score of $>5 \%$, three a fall of $>5 \%$ and all others changed less than this amount. The biggest change was in the Trust that performed worst in the first quarter. Practical problems identified included accurate case identification and case note data recording. Despite this the Advancing Quality programme is a practical way of measuring OMs in pneumonia. Further work is required to generate improvements in patient care.

\section{S127 IS HEALTHCARE ASSOCIATED PNEUMONIA A DISTINCT CLINICAL PHENOTYPE?}

doi:10.1136/thx.2010.150946.28

${ }^{1} \mathrm{~J}$ Taylor, ${ }^{1} \mathrm{~J}$ Chalmers, ${ }^{1} \mathrm{~A}$ Singanayagam, ${ }^{1} \mathrm{~A}$ Akram, ${ }^{2} \mathrm{G}$ Choudhury, ${ }^{2} \mathrm{P}$ Mandal, ${ }^{2} \mathrm{~A}$ Hill. ${ }^{1}$ University of Edinburgh, Edinburgh, UK; ${ }^{2}$ Royal Infirmary of Edinburgh, Edinburgh, UK

Introduction US Guidelines define healthcare associated pneumonia (HCAP) as patients with regular contact with healthcare (eg, nursing home residents, patients with recent hospital admissions or regular outpatient clinics). It is argued that such patients are more likely to develop infection with resistant organisms and require broader spectrum antibiotics. UK guidelines do not distinguish between HCAP and community-acquired pneumonia (CAP). The aim of this study was to investigate whether HCAP is a distinct clinical phenotype.

Methods We studied consecutive patients aged $18+$ presenting to an emergency department with CAP according to current UK definitions (NHS Lothian 2006-2009). Exclusion criteria included hospital acquired pneumonia, immunosupressed patients, and patients not actively treated (palliative). Cases were reviewed by two investigators and HCAP patients were defined according to 2005 ATS/IDSA guidelines. Survival was analysed by Kaplain-Meier analysis and logistic regression.

Results This study analysed 1111 consecutive patients. Of these, $224(20.2 \%)$ met the criteria for HCAP (39.3\% hospitalised within 3 months, $37.5 \%$ nursing home residents, $10.7 \%$ recent outpatient appointments, $12.5 \%$ 'other'). 96.4\% of HCAP patients received standard 'CAP' antibiotic therapy without coverage of Pseudomonas aeruginosa or MRSA. Demographic comparison of HCAP and CAP patients showed HCAP patients were significantly older (median age 76 vs $64, p<0.0001$ ) and more likely to have co-morbidities, for example, congestive cardiac failure ( $30 \%$ vs $17 \%, p<0.0001)$, COPD ( $33.5 \%$ vs $20.8 \%, p<0.0001)$. HCAP patients had higher markers of severity and worse outcomes on univariate analysis. Mean admission CURB65 score was greater (2.32 vs $1.78, \mathrm{p}<0.0001)$, median length of stay was longer ( 7 vs 5 days, $\mathrm{p}=0.01$ ) and 30 -day mortality was double that of CAP patients $(16.5 \%$ vs $8.2 \%, p=0.0004)$. Kaplain-Meier analysis showed higher mortality for HCAP patients (Log rank test $\chi^{2} 13.24 \mathrm{df}=1, \mathrm{p}=0.0003$ ) as shown in Abstract S127 Figure 1. On multivariate analysis, however, after adjustment for age, co-morbidities and initial pneumonia severity, HCAP was not independently associated with increased 30-day mortality AOR 1.13 (0.69-1.84, $\mathrm{p}=0.6)$

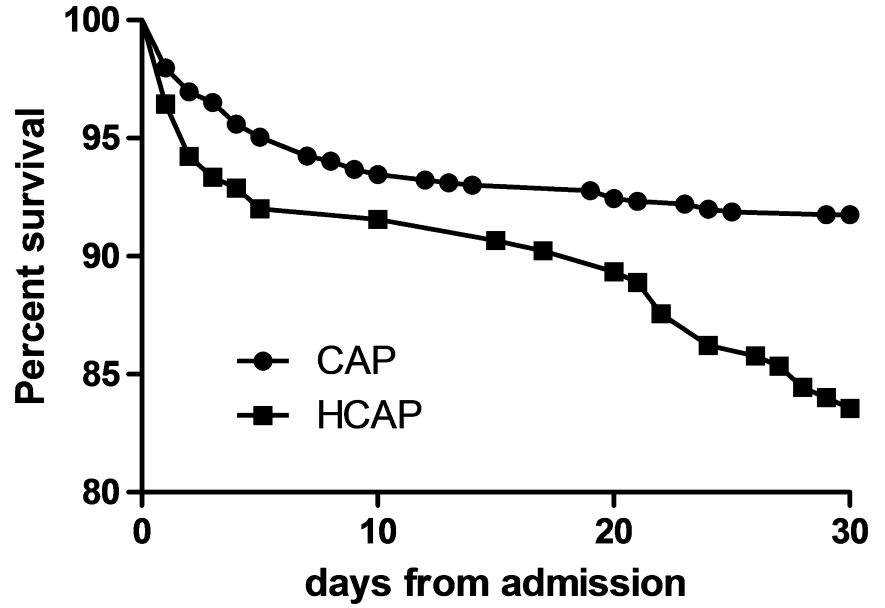

Abstract S127 Figure 1

Conclusions Healthcare associated pneumonia is common, with around $20 \%$ of CAP patients meeting the definition. Although HCAP patients are older and have more co-morbidities, HCAP is not independently associated with 30-day mortality.

\section{S128 ANALYSIS OF VOLATILE BIOMARKERS WITHIN EXHALED BREATH FOR THE DIAGNOSIS OF PNEUMONIA}

doi:10.1136/thx.2010.150946.29

P R Boshier, V Mistry, J R Cushnir, S Curtis, S Elkin, O M Kon, N Marczin, G B Hanna. Imperial College, London, UK

Introduction Whilst pneumonia is a common condition affecting both medical and surgical patients there is currently no definitive diagnostic test. One novel approach may be through the non-invasive analysis of volatile metabolites within exhaled breath, released either by pathogens or host cells. The objective of this study was to delineate the metabolic phenotype of the exhaled breath of patients with community acquired (CAP) and post-operative pneumonia.

Methods Breath samples were collected from patients with a confirmed diagnosis of CAP $(n=26)$. Targeted analysis of six prominent breath metabolites was performed by selected ion flow tubemass spectrometry. Metabolites found to be significantly different in the breath of CAP patients were subsequently investigated in patients undergoing both major abdominal and thoracoabdominal surgery $(n=40)$. Receiver operating characteristic (ROC) analysis of putative pneumonia biomarkers was performed in an independent cohort of patients with suspected CAP $(n=21)$. A diagnosis of pneumonia was established through fulfilment of pre-defined radiological, microbiological, haematological and clinical criteria.

Results Compared to healthy controls, CAP patients had significantly lower breath levels of hydrogen cyanide (6 vs $14 \mathrm{ppb}$, $\mathrm{p}=0.001$ ) and isoprene (72 vs $111 \mathrm{ppb}, \mathrm{p}=0.014$ ). Patients who developed postoperative pneumonia $(n=8)$ had significantly lower levels of hydrogen cyanide within their breath compared to both those patients who had an uncomplicated recovery (4 vs $13 p p b$, $p=0.008)$ and healthy controls. Whilst patients who underwent thoracoabdominal compared to abdominal surgery had higher breath levels of isoprene at both 96 (114 vs $176 \mathrm{ppb}, \mathrm{p}=0.02)$ and $168 \mathrm{~h}$ (119 vs $176 \mathrm{ppb}, \mathrm{p}=0.02)$ following surgery, this did not correlate with the onset of pneumonia. ROC analysis of hydrogen cyanide and isoprene are displayed in Abstract S128 Figure 1. In both medical and surgical patients there was no significant change in the levels of other examined breath metabolites: acetone, ethanol, propanol and acetic acid. 


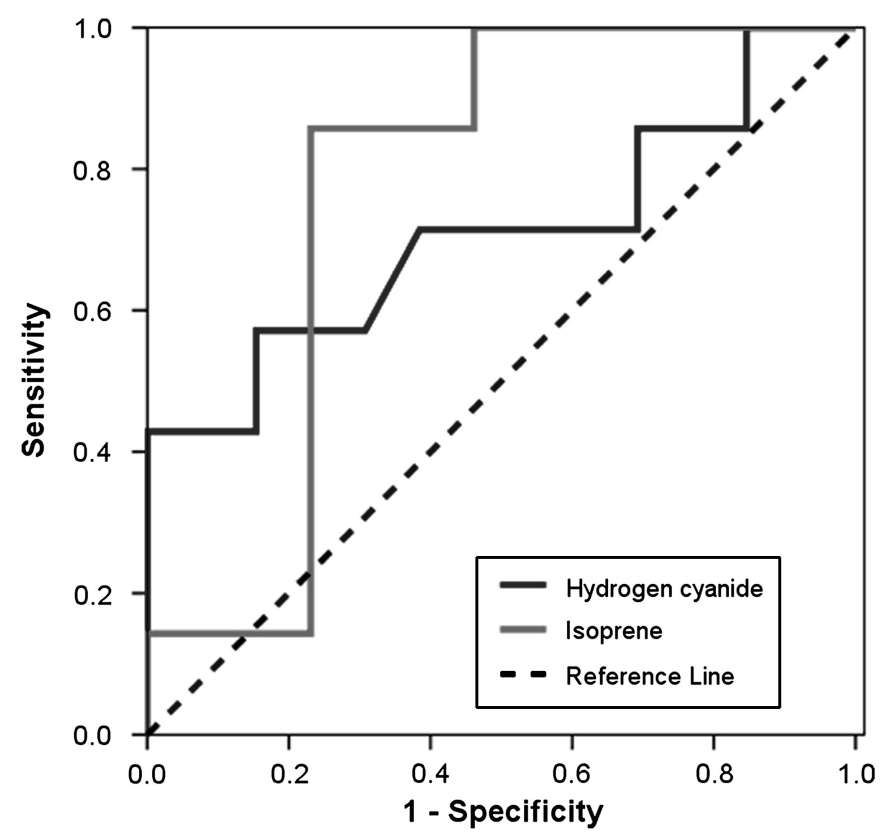

Abstract S128 Figure 1 Receiver operating characteristic (ROC) analysis for hydrogen cyanide and isoprene. Analysis of hydrogen cyanide and isoprene was performed in the exhaled breath of 21 patients with suspected community acquired pneumonia (CAP). A diagnosis of pneumonia was confirmed in 14 patients who met pre-defined criteria for this condition. For patients with suspected CAP and breath hydrogen cyanide $<6.2 \mathrm{ppb}$ sensitivity and specificity were 0.71 and 0.62 respectively (area under ROC curve, 0.71 ). For patients with suspected CAP and breath isoprene $<89 \mathrm{ppb}$ sensitivity and specificity were 0.86 and 0.77 respectively (area under ROC curve, 0.77 ).

Conclusions Results provide evidence that the levels of several prominent breath metabolites, which are potentially related to bacterial activity and lung injury, are significantly altered in patients with this disease. Breath analysis may therefore provide a novel, non-invasive and reliable strategy for the diagnosis of pneumonia.

\section{S129 PREDICTING OUTCOME FROM HIV-ASSOCIATED PNEUMOCYSTIS PNEUMONIA}

doi:10.1136/thx.2010.150946.30

${ }^{1} \mathrm{D}$ Armstrong-James, ${ }^{2} \mathrm{~A} J$ Copas, ${ }^{3} \mathrm{P} \mathrm{D}$ Walzer, ${ }^{4} \mathrm{~S}$ G Edwards, ${ }^{2} \mathrm{R}$ F Miller. ${ }^{1}$ Hospital for Tropical Diseases, London, UK; ${ }^{2}$ University College London, London, UK; ${ }^{3}$ University of Cincinnati, Cincinnati, USA; ${ }^{4}$ Camden Provider Services PCT, London, UK

Background The presentation of Pneumocystis jirovecii pneumonia (PCP) ranges from mild to severe. The former responds to antimicrobial therapy, the latter has a high mortality rate despite treatment. Several studies have described clinical and laboratory factors that are predictive of death from PCP. Our objectives were to create a prognostic scoring model to aid the clinician in predicting outcome from HIV-associated PCP.

Methods A prognostic scoring model was built using risk factors identifiable at/soon after hospitalisation, that is, 'by the bedside'which have previously been identified as being associated with mortality from PCP (a repeat episode of PCP, the patient's age, their haemoglobin $(\mathrm{Hb}), \mathrm{PaO}_{2}$ (breathing room air), both on admission, the presence of co-morbidity (Comorb), such as lymphoma or pregnancy, and the presence of pulmonary Kaposi sarcoma (PKS) (Walzer PD, et al CID 2008;46:625-33). The model was built from data concerning 592 consecutive episodes of PCP that had occurred among 540 patients presenting to a specialist inpatient HIV treatment centre.
Results The prognostic scoring model was: $[25.5+$ (age in years/10) +2 (if a repeat episode of PCP) +4 (if PKS detected) +4 (if Comorb present $)-\mathrm{PaO}_{2}-\mathrm{Hb}$. The prognostic model produced scores ranging from 0 to 20; median (IOR)=9 (7-11). Testing interactions between risk factors and time showed the model to be applicable across all time periods. Patients were divided into five groups according to prognostic score: $0-3.9=$ group $1,4-7.9=$ group 2 , $8-11.9=$ group $3,12-15.9=$ group 4,16 or greater =group 5 . Abstract S129 Table 1 shows mortality rates among the 540 patients with PCP, grouped according to their prognostic scores.

\section{Abstract S129 Table 1}

\begin{tabular}{ll}
\hline Group & Mortality (\%) \\
\hline 1 & 0 \\
2 & 3 \\
3 & 13 \\
4 & 29 \\
5 & 59 \\
\hline
\end{tabular}

Conclusions While this prognostic scoring model requires further validation in patient cohorts from other healthcare institutions, it is potentially a simple 'by the bedside' method of identifying patients early in their hospital admission who are at high and low risk of in-hospital death from PCP and so may aid the clinician in assessing the severity of illness and in deciding on treatment strategies.

\section{S130 OUTCOMES OF PSEUDOMONAS ERADICATION THERAPY IN PATIENTS WITH NON-CYSTIC FIBROSIS BRONCHIECTASIS}

doi:10.1136/thx.2010.150946.31

L White, G Mirrani, M Grover, J Rollason, A Malin, J Suntharalingam. Royal United Hospital, Bath, UK

Introduction and objectives In patients with non-cystic fibrosis (CF) bronchiectasis, Pseudomonas aeruginosa infection is associated with increased hospital admissions, poorer quality of life and accelerated lung function decline. The BTS guidelines recommend early Pseudomonas eradication therapy. However, despite clear evidence in CF, there is little evidence in non-CF bronchiectasis. Since 2004, we have initiated eradication therapy following initial Pseudomonas infection in non-CF bronchiectasis patients. This retrospective study assessed clinical and microbiological outcomes.

Methods All patients undergoing Pseudomonas eradication therapy from 2004 to 2010 were identified from our electronic database. Therapy included intravenous, oral and nebulised antibiotics. Patients were assessed for microbiological eradication, exacerbation frequency (measured by number of antibiotic courses), hospital admissions, clinical symptoms and lung function.

Results 30 patients were identified. $21(67.7 \%)$ were females and mean age was 62.2 years. Median follow-up time was 26.4 months. Eradication therapy involved 2 weeks of intravenous antibiotics (12 patients), intravenous antibiotics followed by oral ciprofloxacin (13 patients) or oral ciprofloxacin alone (five patients), combined with 1-4 months of nebulised colomycin in 26 patients. Pseudomonas was initially eradicated from sputum in 24 patients (80.0\%). 13 patients (43.3\%) remained Pseudomonasfree at latest follow-up. 11 patients $(36.7 \%)$ were subsequently reinfected with median time to reinfection of 6.2 months (Abstract S130 Figure 1). 17 patients with chronic infection were maintained on regular nebulised antibiotics. Exacerbation frequency was significantly reduced, with mean number of antibiotic courses per year 3.93 pre-eradication, and 2.09 post- 